\title{
Vibrations of the cubane molecule: inelastic neutron scattering study and theory
}

\author{
T. Yildirim ${ }^{\text {a,b, * }}$, Ç. Kılıç c ${ }^{\text {, S. Siraci }}{ }^{\text {c }}$, P.M. Gehring ${ }^{\text {b }}$, D.A. Neumann ${ }^{\text {b }}$, \\ P.E. Eaton ${ }^{\mathrm{d}}$, T. Emrick ${ }^{\mathrm{d}}$ \\ ${ }^{a}$ University of Maryland, College Park, MD 20742, USA \\ ${ }^{\mathrm{b}}$ NIST Center for Neutron Research, National Institute of Standards and Technology, Gaithersburg, MD 20899, USA \\ ${ }^{\mathrm{c}}$ Department of Physics, Bilkent University, Bilkent 06533, Ankara, Turkey \\ ${ }^{\mathrm{d}}$ Department of Chemistry, University of Chicago, Chicago, IL 60637, USA
}

Received 4 May 1999; in final form 7 June 1999

\begin{abstract}
Cubane $\left(\mathrm{C}_{8} \mathrm{H}_{8}\right)$ is an immensely strained molecule whose $\mathrm{C}-\mathrm{C}-\mathrm{C}$ bond angle is $90^{\circ}$ rather than $109.5^{\circ}$ as expected for $\mathrm{sp}^{3}$ bonding of carbon. We have measured the intramolecular vibrational spectrum of cubane using inelastic neutron scattering. The neutron data are used to test the transferability of various phenomenological potentials and tight-binding models to this highly strained molecule. Unlike these models, first-principles calculations of the INS spectrum (both energy and intensity) agree well with the experimental data. (C) 1999 Published by Elsevier Science B.V. All rights reserved.
\end{abstract}

\section{Introduction}

Cubane $\left(\mathrm{C}_{8} \mathrm{H}_{8}\right)[1,2]$ is an atomic scale realization of a cube. This intriguing molecular geometry imposes an angle of $90^{\circ}$ on the $\mathrm{C}-\mathrm{C}-\mathrm{C}$ bond instead of the $109.5^{\circ}$ normally found for $\mathrm{sp}^{3}$-bonding of carbon. Cubane therefore possesses a tremendous amount of strain energy, roughly $6.5 \mathrm{eV} /$ molecule $(150 \mathrm{kcal} / \mathrm{mol})$. The cubic geometry has been confirmed using a wide variety of experimental techniques including high-resolution laser [3], infrared (IR) and Raman spectroscopy [4-6], X-ray diffraction [7] and ${ }^{13} \mathrm{C}$ NMR measured in the solid phase

\footnotetext{
* Corresponding author. NIST Center for Neutron Research, National Institute of Standards and Technology, Gaithersburg, MD 20899, USA. Fax: + 1-301-921-9847; e-mail: taner@nist.gov
}

[8], as well as by a number of theoretical studies [9-12] performed on both a semi-empirical and ab initio level. More recent work has centered on the structural and dynamical properties of solid cubane and other cubane-based compounds [14-19]. These studies have demonstrated the existence of a solidsolid phase transition in cubane and have characterized the structure of the high-temperature phase as well as the central role played by the dynamics in this transition.

Because of the unique bonding geometry of cubane, it is important to understand its vibrational spectrum. It has already been studied by Raman and IR spectroscopy [4-6]. However, even though the resolutions of these techniques are quite good (of order $0.2 \mathrm{meV}$, compared to $1-10 \mathrm{meV}$ in neutron scattering), they can only probe $\mathbf{q}=0$ modes and they are also subject to selection rules. Therefore 
information from such techniques is limited. Here we present a complementary approach, inelastic neutron scattering (INS), which probes the modes at all q-values without any selection rules. Hence the scattered neutron intensity contains valuable information about the eigenvectors of the vibrations. One can test various potential models by comparing the experimental INS spectrum with not only the mode energies, but also the eigenmode intensities, which are more difficult to predict.

In recent years, there has been a growing interest in developing simple but efficient force-field models to predict the dynamics and vibrational spectra of a wide range of systems [20]. However, these models have mostly been tested using standard molecules such as methane, octane, etc. It is therefore of interest to see how well these models would work for a very unusual and highly strained system such as cubane.

In Section 2, we discuss the experimental setup, the relationship of the vibrational eigenmodes to the neutron scattering intensity, and the observed spectrum in terms of vibrations of the cubane molecule. In Section 3, we calculate the INS spectrum using three different approaches: (1) a widely used phenomenological potential, (2) a transferable tight-binding model, and (3) first-principles calculations. Comparison of the spectra obtained from these models to the measured spectrum will provide information about the accuracy of these techniques and their transferability (i.e. applicability) to unusually strained systems. Our results will be summarized in Section 4.

\section{Vibrational spectrum and inelastic neutron scattering}

The cubane molecule $\left(\mathrm{C}_{8} \mathrm{H}_{8}\right)$ has $3 \times(8+8)=$ 48 degrees of freedom, three of which are translational modes of the molecule, and three of which correspond to rotations of the molecule. Thus there are 42 internal degrees of freedom and 42 individual vibrational eigenmodes. Because of the highly symmetric structure of cubane, these eigenmodes have only 18 distinct frequencies; i.e. $2 \times(2 \mathrm{~A}+5 \mathrm{~T}+2 \mathrm{E})$. The only IR active modes are the three $\mathrm{T}_{1 \mathrm{u}}$ modes, and the observation of just three strong bands in the
IR spectrum [5] confirms the $\mathrm{O}_{\mathrm{h}}$ symmetry of the cubane molecule. The Raman active modes are the two $A_{1 g}$ modes, the two $E_{g}$ modes, and the four $T_{2 g}$ modes. This leaves 15 so-called 'silent' modes: two $A_{2 u}$, two $E_{u}$, one $T_{1 g}$, and two $T_{2 u}$. Any model description of the intramolecular force field of cubane must successfully predict the frequencies of all of the modes. Thus it is important to determine the frequencies of the silent modes experimentally. Several approaches have been employed to accomplish this, including observations of weak peaks in IR and Raman spectra due to ${ }^{13} \mathrm{C}$ impurities, crystal fields, and combination modes [4-6]. Neutron spectroscopy is perhaps the most useful of these methods since it is not subject to any selection rules [21].

Within the independent molecule and incohorent approximations [21], the observed quantity for onephonon scattering in neutron energy loss may be written as [21]:

$$
\begin{aligned}
& \frac{\mathrm{d}^{2} \sigma}{\mathrm{d} \Omega \mathrm{d} E} \\
& =\frac{N}{8 \pi} \frac{k_{f}}{k_{i}}[n(\omega)+1] \frac{Q^{2}}{\omega} \sum_{j=1}^{3 N-6} \delta\left(\omega-\omega_{j}(\vec{q})\right) \\
& \quad \times \sum_{i=1}^{N} \frac{\sigma_{i}}{M_{i}}\left|\hat{Q} \cdot \hat{e}_{i j}\right|^{2} \mathrm{e}^{-2 W_{i}(\vec{Q})},
\end{aligned}
$$

where $\sigma_{i}, M_{i}$, and $\mathrm{e}^{-2 W_{i}(\vec{Q})}$, are the total bound scattering cross-section, the mass, and the DebyeWaller amplitude of atom $i$, respectively, and $\hat{e}_{i j}(\vec{q})$ is its eigenvector component, and $\vec{r}_{i}$ is its position. Here, $k_{i}$ and $k_{f}$ are the initial and final neutron wave vectors, respectively. $N$ is the total number of molecules and $n(\omega)$ is the Bose factor. To the extent that $k_{f}$ is much smaller than $k_{i}$ (which is normally the case for the type of spectrometer used in our measurements) $Q^{2} / \omega$ is approximately independent of $\omega$. Then the observed intensity in a low-temperature experiment is approximately proportional to the phonon density of states. The averaging of $\left|\hat{Q} \cdot \hat{e}_{i j}\right|^{2}$ occurs within the region of $\vec{Q}$-space sampled by the spectrometer when it is set to detect neutrons whose energy transfer is $\hbar \omega$, and it includes an average over $\hat{Q}$ if the sample is a powder.

INS measurements were performed using the filter analyzer spectrometer located on beamline BT4 
at the NIST Center for Neutron Research [22]. In most of the measurements, a $\mathrm{Cu}(220)$ monochromator, surrounded by $60^{\prime}-40^{\prime}$ horizontal collimation and combined with a cooled polycrystalline beryllium filter analyzer was used. The relative energy resolution of this instrument was approximately $8 \%$ in the energy range probed. The sample (about $0.5 \mathrm{~g}$ of cubane powder) was held at $10 \mathrm{~K}$ within a helium-filled flat aluminum can using a closed-cycle He refrigerator.

The measured inelastic scattering spectrum is shown in Fig. 1. The lowest observed peak is at an energy of $75 \mathrm{meV}$, almost six times higher than that of the highest energy lattice mode [14]. The inset to Fig. 1 shows four particular modes which are identified from first-principles calculations (see discussion below). The lowest energy mode $\left(E_{\mathrm{u}}\right)$ is the one where two opposing faces of the cubane molecule twist with respect to each other. The second lowest mode $\left(T_{2 \mathrm{~g}}\right)$ at $82.5 \mathrm{meV}$ is one where the square shape of two opposing faces is distorted into a diamond shape. At high energies, the resolution is not good enough to resolve the multiple peaks. Based on the assignment from first-principles calculations we show two such high-energy modes in the right two figures of the inset to Fig. 1. It is interesting to note that the highest H-bending frequency is significantly lower than for most other hydrocarbons.

\section{Theory}

We now turn our attention to calculations of the normal modes of cubane. These have been performed using three different approaches: a widely used phenomenological potential, a transferable tight-binding model, and a first-principles calculation. Table 1 summarizes the equilibrium structure and the $\mathbf{q}=0$ vibrational energies obtained from the models as well as the experimental values.

\subsection{Empirical potential models}

Despite rapid developments in computers and computational techniques, empirical potentials are still widely used in a broad range of systems due to a need for large-scale simulations. Hence, it is interesting to know how well the empirical potentials work for a molecule as highly strained as cubane.

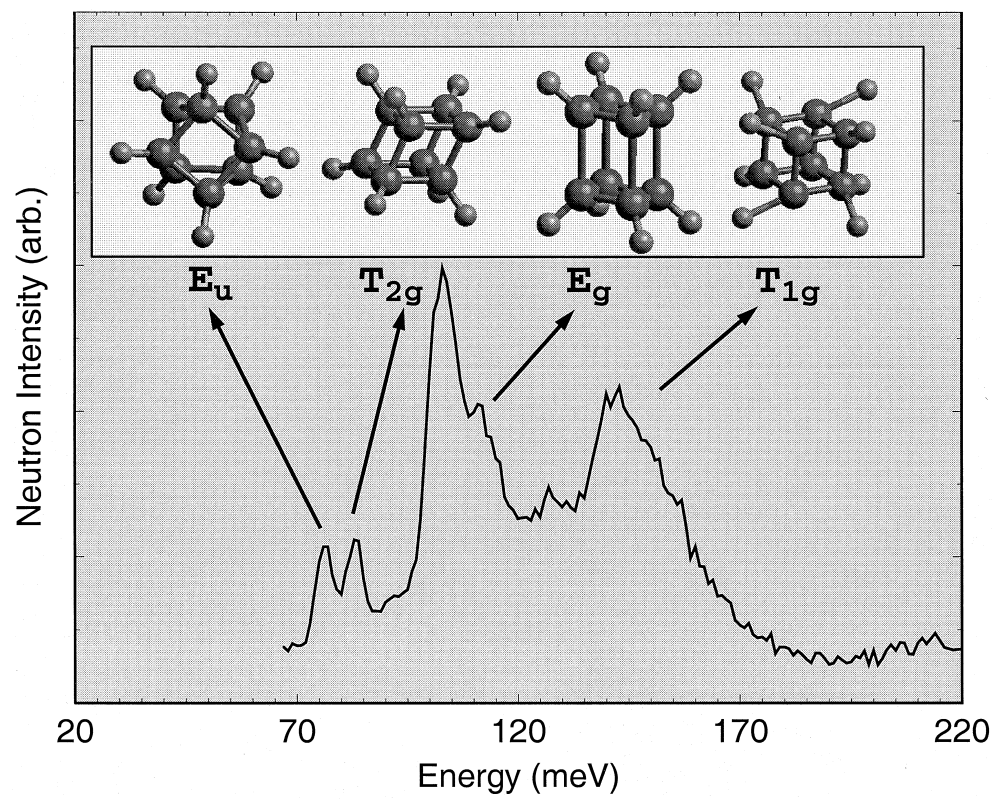

Fig. 1. Measured inelastic scattering spectrum of solid cubane. The peaks are labeled according to the first principles calculations (see below). The inset shows four typical vibrational modes at different energies. Animated movies of these modes can be found at the web site: http://rrdjazz.nist.gov/ taner/cubanehome.html 
Table 1

Vibrational mode energies of cubane (in meV) from model calculations (see text) and measurements. The left column indicates the symmetry of the modes and the Raman (R) and IR activities. The symbols COM, TB, 6-31G, and DNP correspond to respectively the phenomenological potential model COMPASS, tight-binding model, first principles calculations with GAUSSIAN 94 (using 6-31G basis) [27,28] and DMOL (using DNP basis) [27,29]. The last row indicates average percentage error per mode, which is defined as $\frac{1}{N} \sum \frac{\sum d_{\mathrm{o}}\left|\omega_{\mathrm{o}}-\omega_{\mathrm{c}}\right|}{\omega_{\mathrm{o}}}$, where $\omega_{\mathrm{o}}, \omega_{\mathrm{c}}$ are the observed and calculated mode energies, respectively, and $d_{\mathrm{o}}$ is the degeneracy. There are a total of $N=42$ modes

\begin{tabular}{|c|c|c|c|c|c|}
\hline Quantity & $\mathrm{COM}$ & $\mathrm{TB}$ & $6-31 \mathrm{G}$ & DNP & Exp. $[4,5]$ \\
\hline$\overline{d_{\mathrm{C}-\mathrm{C}}}$ & 1.538 & 1.678 & $\overline{1.568}$ & 1.571 & $1.562[23]$ \\
\hline$d_{\mathrm{C}-\mathrm{H}}$ & 1.102 & 1.089 & 1.105 & 1.096 & $1.097[23]$ \\
\hline$\overline{A_{1 g}(R)}$ & 385.4 & 404.3 & 384.1 & 380.2 & 371.3 \\
\hline $\mathrm{T}_{1 \mathrm{u}}(\mathrm{IR})$ & 384.6 & 401.7 & 381.1 & 377.8 & 369.2 \\
\hline $\mathrm{A}_{2 \mathrm{u}}$ & 384.6 & 400.0 & 378.6 & 375.6 & 369.2 \\
\hline $\mathrm{T}_{2 \mathrm{~g}}(\mathrm{R})$ & 384.4 & 400.4 & 379.9 & 377.0 & 368.2 \\
\hline $\mathrm{T}_{1 \mathrm{u}}(\mathrm{IR})$ & 166.6 & 170.0 & 152.6 & 151.3 & 152.5 \\
\hline $\mathrm{T}_{2 \mathrm{~g}}(\mathrm{R})$ & 162.1 & 155.2 & 146.4 & 144.5 & 146.6 \\
\hline $\mathrm{E}_{\mathrm{u}}$ & 150.2 & 152.6 & 141.3 & 139.7 & 142.7 \\
\hline $\mathrm{T}_{1 \mathrm{~g}}$ & 151.6 & 145.3 & 138.1 & 138.1 & 140.1 \\
\hline$E_{g}(R)$ & 124.5 & 150.4 & 138.0 & 133.4 & 134.3 \\
\hline $\mathrm{T}_{2 \mathrm{u}}$ & 134.8 & 142.5 & 131.1 & 126.9 & 128.4 \\
\hline$A_{1 g}(R)$ & 122.4 & 171.3 & 129.7 & 123.3 & 124.2 \\
\hline$E_{g}(R)$ & 110.0 & 114.3 & 115.1 & 110.0 & 113.1 \\
\hline $\mathrm{T}_{1 \mathrm{u}}^{\mathrm{g}}(\mathrm{IR})$ & 111.4 & 117.7 & 108.5 & 103.7 & 105.8 \\
\hline$A_{2 u}$ & 140.6 & 149.0 & 117.3 & 125.5 & 104.0 \\
\hline $\mathrm{T}_{2 \mathrm{u}}$ & 110.7 & 106.3 & 104.7 & 100.2 & 102.8 \\
\hline $\mathrm{T}_{2 \mathrm{~g}}(\mathrm{R})$ & 102.0 & 100.0 & 103.4 & 99.9 & 101.8 \\
\hline $\mathrm{T}_{2 \mathrm{~g}}(\mathrm{R})$ & 76.4 & 97.2 & 80.1 & 80.7 & 82.5 \\
\hline $\mathrm{E}_{\mathrm{u}}$ & 64.4 & 80.3 & 72.7 & 73.7 & 76.5 \\
\hline$\%$-error & $3.28 \%$ & $4.93 \%$ & $2.19 \%$ & $2.13 \%$ & -- \\
\hline
\end{tabular}

We have therefore calculated the optimized structure and the molecular vibrations of cubane using an empirical potential model called 'COMPASS' [24]. Like many force-field models, the functionals used in COMPASS have valence and non-bonding interaction terms. The valence terms represent the internal coordinates of the bond lengths $(b)$, bond angles $(\theta)$, torsion angle $(\phi)$, etc. The cross-terms of two or three internal coordinates are also included in COMPASS, and play an important role in predicting the vibrational frequencies. The non-bonding terms are the Coulomb and van der Waals interactions. Details of the potential and the numerical values of the parameters used in COMPASS can be found in Ref. [24]. The results obtained from this potential using all the terms are summarized in Table 1. The bond lengths as well as the frequencies agree very nicely with the experimental data. However, despite the large number of parameters and terms in this potential model, the agreement of the calculated spectrum with the measured one is not good enough to make an unambiguous assignment of the modes (Fig. 2).

We have also calculated the INS spectrum using only the bond stretching and bond bending terms in COMPASS. The result was similar to that obtained using all terms except that there was only one feature below $90 \mathrm{meV}$. We then added cross-terms one at a time, obtaining the best agreement when the torsion-bend-bend term is included in the potential. Thus, the potential model with the minimum number of terms has the form:

$$
\begin{aligned}
E= & \sum_{b, n=2,3,4} k_{n}^{r}\left(r^{b}-r_{\mathrm{o}}^{b}\right)^{n} \\
& +\sum_{a, n=2,3,4} k_{n}^{a}\left(\theta^{a}-\theta_{\mathrm{o}}^{a}\right)^{n} \\
& +\sum_{a, a^{\prime}, \phi} k\left(\theta^{a}-\theta_{0}^{a}\right)\left(\theta^{a^{\prime}}-\theta_{0}^{a^{\prime}}\right) \cos (\phi)
\end{aligned}
$$




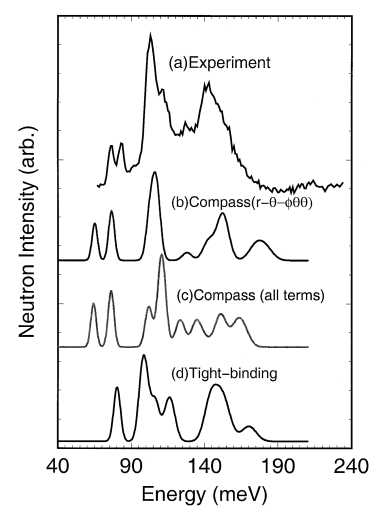

Fig. 2. Comparison of the (a) experimental INS data of cubane with spectra calculated using (b) the generic empirical potential model COMPASS [24] with only stretch $(r)$, bend $(\theta)$, and a cross-term torsion-bend-bend $(\phi-\theta-\theta)$, (which is essential to produce the lowest two modes), and (c) with all terms, and (d) a tight-binding model developed for hydrocarbons $[25,26]$.

The INS spectrum obtained using only these three terms is also shown in Fig. 2. The cross-term is essential to reproduce the lowest energy modes below $90 \mathrm{meV}$. The overall agreement with the intensities is fair, but it is still not good enough to make an unambiguous assignment of the modes.

\subsection{Tight-binding model}

The interest in semi-empirical models is due to their relatively accurate description of a wide range of systems without the time and computational efforts required of first-principles calculations. For hydrocarbons, where the covalent bonds are very strong and directional, it has been shown that a simple minimum basis tight-binding Hamiltonian works very well for both the structural and vibrational properties of a wide variety of systems $[25,26]$. In these models, the tight-binding Hamiltonian usually includes only the $2 \mathrm{~s}$ and $2 \mathrm{p}$ valence electrons of carbon and the $1 \mathrm{~s}$ electron of hydrogen, and has the form:

$H=\sum_{\alpha, i} \varepsilon_{\alpha}^{i} a_{\alpha, i}^{+} a_{\alpha, i}+\sum_{\alpha, \beta, i, j} t_{\alpha, \beta}^{i j}\left(r_{i j}\right) a_{\alpha, i}^{+} a_{\alpha, j}$,

where $i$ and $j$ label the atoms and $\alpha$ and $\beta$ label the atomic orbitals. $\varepsilon_{\alpha}^{i}$ is the atomic orbital energy of atom $i$ and orbital $\alpha[25,26]$.
The cohesive energy of the system is defined as

$$
E_{\mathrm{coh}}=E_{\mathrm{val}}+\sum_{i<j} E_{\mathrm{core}}^{i j}\left(r_{i j}\right)-\sum_{i} E_{\mathrm{atom}}^{i},
$$

where $E_{\mathrm{val}}$ is the sum of electronic eigenvalues over all occupied electronic states, $E_{\text {core }}^{i j}$ (the core repulsion energy) is the screened ion-ion interaction between atom $i$ and $j$, and $E_{\text {atom }}^{i}$ (the atomic energy) is the reference energy of the isolated atom $i$ in the dissociation limit.

The electronic hopping matrix elements $t_{\alpha, \beta}(r)$ and the core repulsive interactions $E_{\text {core }}(r)$ have been parameterized for their distance dependence [25]. Wang and Mak tested the transferability of this model to a large number of hydrocarbons by comparison to both ab initio calculations and experimental data [25]. The model correctly reproduced changes in the electronic configuration as a function of the local bonding geometry around each carbon atom. We have therefore calculated the vibrational spectrum of cubane using this model without any adjustments. From Table 1 it is clear that the overall agreement with the experimental vibrational mode energies is reasonable. However, as was the case for the empirical potential models, the tight-binding model does not reproduce the features observed in the INS spectrum well enough to identify the modes. The biggest failure occurs for the lowest mode, which is missing in the tight-binding model. We believe that we could not significantly improve this result simply by changing the values of the parameters in the tight-binding model. This is because the interactions in the model are pair-wise and, as we showed previously, a three-body type of interaction is needed to reproduce the lowest doublet in the observed spectrum.

\subsection{First-principles calculations}

We have also calculated the vibrational spectrum of a cubane molecule using density-functional theory. This has been done using two different approaches, GAUSSIAN 94 [27,28] and DMOL [27,29] in order to examine the efficiency and accuracy of different type of basis sets used in DFT within the local density approximation (LDA).

The DMOL calculation was carried out using a first-principles density-functional approach with analytic energy gradients [27,29]. The Hedin-Lundqvist 
form was used for the exchange-correlation energy of the electron within the LDA. The calculation was performed with a double-numerical basis set augmented with polarization (DNP). Geometry optimization was carried out using the conjugate-gradient technique. The dynamical matrix was obtained by calculating the forces exerted on all the atoms in the molecule when one atom is displaced in the $x, y$, and $z$ directions by a distance of $0.03 \AA$. Both positive and negative displacements were considered to minimize the effects of anharmonicity. In GAUSSIAN 94 calculations, we used the $6-31 \mathrm{G}$ basis $[31,32]$ with a Slater local spin density exchange (i.e. $\rho^{2 / 3}$ with the theoretical coefficient of 2/3) and a Perdew's gradient-corrected local correlational functional [30].

For both the geometry optimization and frequency calculations, we used the full point group symmetry of cubane molecule, which is $\mathrm{O}_{\mathrm{h}}$. Hence, there are only two degrees of freedom, namely the $\mathrm{C}-\mathrm{C}$ and $\mathrm{C}-\mathrm{H}$ bond lengths. The optimized values of the bond lengths and the energies of the modes are once again summarized in Table 1. Both GAUSSIAN 94 and DMOL give results that are in excellent agreement with the experimental values.

Since these calculations are for the gas phase, the agreement with the experiments indicates that the dispersion of the intramolecular phonons are very small, and therefore negligible. The rigidity of the cubane molecule suggests such that the vibrational properties in the gas phase and in the solid phase

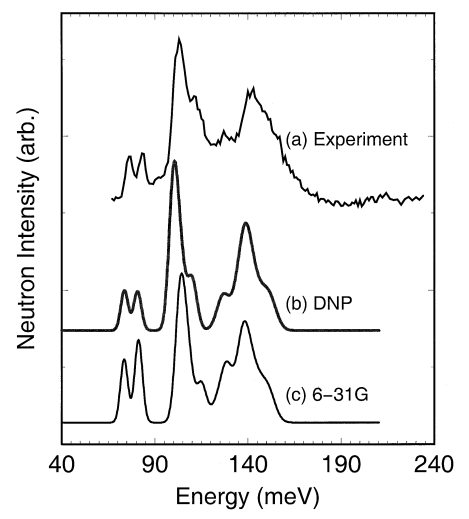

Fig. 3. Comparison of the (a) experimental INS spectrum of Cubane with two different first-principles calculations; (b) DMOL and (c) GAUSSIAN 94. must be nearly identical. We are carrying out similar calculations in the solid state to confirm this [33].

In addition to bond lengths and the vibrational mode energies, first-principles calculations also predict the correct eigenmodes for cubane considerably more accurately than do the other calculations reported here. This is evident from the excellent agreement between the calculated INS spectrum and the experimental one (Fig. 3).

As a final remark we note that all of the calculations predict a considerably higher frequency for the $\mathrm{A}_{2 \mathrm{u}}$ mode (Table 1) than is currently accepted. This could be an indication that the assignment of this mode is incorrect. This is quite likely since the IR or Raman intensity of the $A_{2 u}$ mode arises solely from the very small distortion of cubane away from cubic symmetry [4].

\section{Conclusions}

We have studied the molecular vibrations of cubane using INS. The experimental INS spectrum is compared with a large number of calculations based on a generic phenomenological potential, a transferable tight-binding model, and density-functional theory within the local density approximation. Our conclusions are as follows.

(1) The observed intramolecular vibrations of solid cubane are well separated from the lattice modes. The lowest internal mode is observed around 75 $\mathrm{meV}$, which is six times larger than the highest librational energies.

(2) Comparison of the data with a recent empirical potential model indicated that, even though good agreement can be obtained for the phonon energies, it is not possible to explain the intensities of the modes observed in the spectrum. Within this model, the best description of the data is obtained using only a stretching term, a bending term, and a single essential cross-term, namely the tortion-bendingbending term. This potential with three terms is certainly sufficient to describe most of the intramolecular vibrations of cubane in a molecular dynamics simulation.

(3) A transferable tight-binding model developed for hydrocarbons was tested. The accuracy of the 
results for the INS spectrum lie between those obtained from the purely empirical and first-principles calculations.

(4) First-principles calculations give results that agree with the data very nicely, and allowed us to identify the modes observed in the spectrum unambiguously. For instance, the lowest energy intramolecular vibrations of cubane is found to be the one where opposite faces of the cube rotate with respect to each other.

(5) Comparison between calculated and experimental frequencies indicate that, regardless of the method used, the calculated $\mathrm{A}_{2 \mathrm{u}}$ mode energy is much higher than the experimental value. This may be due to an incorrect assignment rather than a failure of the calculations.

\section{Acknowledgements}

The authors acknowledge partial supports from the National Science Foundation under Grant No. INT97-31014 and TÜBITAK under Grant No. TBAG-1668(197 T 116).

\section{References}

[1] P.E. Eaton, T.W. Cole Jr., J. Am. Chem. Soc. 86 (1964) 962.

[2] P.E. Eaton, Angew. Chem. 31 (1992) 1421.

[3] A.S. Pine, A.G. Maki, A.G. Robiette, B.J. Krohn, J.K.G. Watson, Th. Urbanek, J. Am. Chem. Soc. 106 (1984) 891.

[4] E.W. Della, E.F. McCoy, H.K. Patney, G.L. Jones, F.A. Miller, J. Am. Chem. Soc. 101 (1979) 7441.

[5] T.W. Cole, J. Perkins, S. Putnam, P.W. Pakes, H.L. Strauss, J. Phys. Chem. 85 (1981) 2185.

[6] R.A. Dalterio, F.J. Owens, Sol. State Commun. 67 (1988) 673.

[7] E.B. Fleischer, J. Am. Chem. Soc. 86 (1964) 3889.
[8] J.C. Facelli, A.M. Orendt, M.S. Solum, G. Depke, D.M. Grant, J. Michl, J. Am. Chem. Soc. 108 (1986) 4268.

[9] J.M. Schulman, C.R. Fischer, P. Solomon, T.J. Venanzi, J. Am. Chem. Soc. 100 (1978) 2949.

[10] W. Scamehorn, M. Yoshimine, J. Pacansky, J. Phys. Chem. 85 (1981) 1340.

[11] J. Almlof, T. Jonvik, Chem. Phys. Lett. 92 (1982) 267.

[12] C.A. Scamehorn, S.N.M. Hermiller, R.M. Pitzer, J. Chem. Phys. 84 (1986) 833.

[13] S. Borman, Chem. Sci. Eng. News 72 (1994) 34.

[14] P.M. Gehring, D.A. Neumann, W.A. Kamitakahara, J.J. Rush, P.E. Eaton, D.P. VanMeurs, J. Phys. Chem. 99 (1995) 4429.

[15] M.A. White, R.E. Wasylishen, P.E. Eaton, Y. Xiong, K. Pramod, N. Nodari, J. Phys. Chem. 96 (1992) 421.

[16] A. Detken, H. Zimmermann, U. Haeberlen, R. Poupko, Z. Luz, J. Phys. Chem. 100 (1996) 9598.

[17] T. Yildirim, P.M. Gehring, D.A. Neumann, P.E. Eaton, T. Emrick, Phys. Rev. B 60 (1999) 314.

[18] T. Yildirim, P.M. Gehring, D.A. Neumann, P.E. Eaton, T. Emrick, Carbon 36 (1998) 809.

[19] T. Yildirim, P.M. Gehring, D.A. Neumann, P.E. Eaton, T. Emrick, Phys. Rev. Lett. 78 (1997) 4938.

[20] A.J. Pertsin, A.I. Kitaigorodsky (Eds.), The Atom-Atom Potential Method, Springer, 1986.

[21] See, e.g., S. Lovesey, Theory of Neutron Scattering from Condensed Matter, 3rd edn., Oxford University Press, New York, 1987.

[22] J.R.D. Copley, D.A. Neumann, W.A. Kamitakahara, Can. J. Phys. 73 (1995) 763.

[23] Lise Hedberg, K. Hedberg, P.E. Eaton, N. Nodari, A.G. Robiette, J. Am. Chem. Soc. 113 (1991) 1514.

[24] H. Sun, J. Phys. Chem. B 102 (1998) 7338.

[25] Y. Wang, C.H. Mak, Chem. Phys. Lett. 235 (1995) 37.

[26] C.H. Xu, C.Z. Wang, C.T. Chan, K.M. Ho, J. Phys. Condens. Matter 4 (1992) 6047.

[27] Identification of commercial products does not imply recommendation or endorsement by the National Institute of Standards and Technology.

[28] M.J. Frisch et al., GAUSSIAN 94, Gaussian, Inc., Pittsburgh, PA, 1995.

[29] DMOL, Biosym/MSI, San Diego, CA, 1995.

[30] J.P. Perdew, A. Zunger, Phys. Rev. B 23i (1981) 5048.

[31] M.S. Gordon, Chem. Phys. Lett. 76 (1980) 163

[32] W.J. Hehre, R. Ditchfield, J.A. Pople, J. Chem. Phys. 56 (1972) 2257.

[33] T. Yildirim et al., to be published. 